\title{
TIPOLOGÍA DE UNIDADES DE SÍNTESIS DEFINIDAS POR CRITERIOS ECOLÓGICOS QUE PUEDEN ADOPTARSE EN LOS PROCESOS DE MESO ZONIFICACIÓN ECOLÓGICA ECONÓMICA. UNA APROXIMACIÓN AL TERRITORIO PERUANO
}

\author{
TYPOLOGY OF SYNTHESIS UNITS DEFINED BY ECOLOGICAL CRITERIA \\ THAT CAN BE USED IN THE PROCESS OF MESO ECOLOGICAL AND \\ ECONOMIC ZONING. AN APPROACH TO THE PERUVIAN TERRITORY
}

\author{
Santiago Madrigal Martínez ${ }^{1}$
}

\begin{abstract}
Resumen
La clasificación de unidades de síntesis definidas por criterios ecológicos propuesta en este artículo, pretende ser de ayuda en los procesos de meso Zonificación Ecológica Económica en el territorio peruano. Para la tipificación se han empleado las variables de hidrografía, vegetación y fauna silvestre. El procedimiento metodológico desarrollado para definir las unidades se compone de cinco etapas: (I) Uso de la información temática correspondiente a las tres variables; (II) Análisis de cada variable según su criterio dominante; (III) Caracterización de los sistemas; (IV) Caracterización de los sub-sistemas; y (V) Obtención de las unidades de síntesis.

Se han caracterizado ocho sistemas: tres asociados a la hidrografía, dos a la vegetación y otros tres a la fauna silvestre. De igual manera estos sistemas incluyen un total de veinte sub-sistemas, que se desagregan a su vez en cuarenta y tres unidades de síntesis.

Considerando la complejidad del territorio la tipología propuesta intenta ser flexible y estar abierta a nuevas interpretaciones y ampliaciones con nuevas unidades que no se hayan considerado en esta aproximación. Por lo tanto, la categorización realizada no es excluyente.

Palabras clave: unidad de síntesis, unidad territorial, unidad ecológica económica, zonificación ecológica económica, ordenamiento territorial, planificación territorial, Perú, ecorregiones.
\end{abstract}

\begin{abstract}
The ecological classification of synthesis units proposed in this article is intended to be helpful in the process of Meso Ecological Economic Zoning in the Peruvian territory. The typology uses the following variables: hydrography, vegetation and wildlife. The methodological approach developed to define the units has five stages: (I) use of thematic information regarding hydrography, vegetation and wildlife; (II) analysis of each variable according to its dominant criterion; (III) characterization of the systems; (IV) characterization of sub-systems; and (V) obtaining of the synthesis units.

Eight systems have been characterized: three of them associated with hydrography, two with vegetation and three with wildlife. Similarly, these systems include a total of twenty sub-systems, which are disaggregated into forty-three synthesis units.

Considering the complexity of the territory, the typology proposed tries to be flexible and open to new interpretations and extensions adding new units that were not considered in this approach. Thus, proposed classification done is not exclusive.
\end{abstract}

Key words: synthesis unit, land unit, ecological economic unit, ecological economic zoning, land management, land planning, Peru, eco-regions.

\section{Introducción.}

La importancia de los valores ecológicos que contiene el territorio peruano, hace necesaria la aplicación del ordenamiento territorial para promover y facilitar la gestión responsable de la diversidad biológica, la conservación de los ecosistemas y la preservación del patrimonio natural para el bienestar y la salud de la población.
A partir del año 2004, los procesos de ordenamiento territorial en el Perú se instrumentalizan a través del Reglamento de Zonificación Ecológica Económica (DS No 087-2004-PCM). El principal objetivo de este proceso es el análisis del territorio para la evaluación de sus potencialidades y limitaciones con criterios físicos, biológicos, ambientales, sociales, económicos y culturales. 
La Directiva sobre "Metodología para la Zonificación Ecológica y Económica (ZEE)”, en el capítulo 2, apartado 2.2, indica lo siguiente sobre la Meso ZEE:

"[...]. Se aplica a nivel regional y a nivel de provincias y distritos, cuencas hidrográficas y otros ámbitos espaciales con superficies relativamente no muy grandes, incluyendo el área de influencia de zonas metropolitanas, delimitando unidades espaciales del territorio a semi-detalle, con criterios biofísicos y socioeconómicos.

La cartografía aplicable a los estudios del medio biofísico (grandes ecosistemas y paisajes) corresponde a una escala de trabajo igual a 1:100 000. Las unidades espaciales para la información socioeconómica deben corresponder a los distritos o microcuencas."

La ZEE, según Directiva, se compone de cinco etapas, y es concretamente en la segunda donde se da la fase de recopilación, sistematización y generación de información temática. En esta fase se conforma el inventario de las variables abióticas (geología, geomorfología, suelo, hidrografía y clima), las bióticas (vegetación y fauna) y sus consiguientes mapas temáticos. Mediante estas variables se realiza la integración en unidades territoriales en función de ciertos criterios, como son el ecológico, el productivo, el histórico cultural, etc. Estas unidades pueden obtenerse de diferentes métodos, siendo el de unidad de síntesis el elegido en este artículo para la tipificación, el cual se explica más adelante.

Las unidades territoriales en la ZEE son denominadas unidades ecológicas y económicas. Estas también son conocidas como unidades ambientales, que corresponden a áreas con cierta homogeneidad interna definidas por los caracteres más importantes físicos, biológicos y socioeconómicos inventariados en el territorio de estudio (González Bernáldez, 1973).

La unidad territorial está conformada por diferentes variables que se interrelacionan entre sí, y a su vez con el resto de unidades que componen el total del territorio. Por lo tanto, una unidad del territorio es un conjunto de relaciones tangibles tanto internas como externas (Zonneveld, 1989).

El método más utilizado para la obtención de estas unidades es por superposición de los mapas temáticos. El patrón visual en un mapa revela mucho del aspecto posicional y ayuda así en la comprensión de las unidades como cuerpos tangibles (McHarg, 1969). No obstante, este proceso tiene el inconveniente de generar un número excesivo de polígonos ${ }^{1}$ cuando se dispone de muchos mapas o capas temáticas (VV.AA., 2000). Asimismo, esto puede conllevar a errores debido a la excesiva inclusión de variables que apenas aportan información (Ramos Fernández, 1979).

\footnotetext{
1 Clase de entidad utilizada en los sistemas de información geográfica para la representación espacial de áreas.
}

Para evitar este inconveniente se pueden plantear dos formas alternativas, la primera desarrollada por Hills (1961) y que consiste en el método de divisiones sucesivas, que se fundamenta en delimitar en la primera etapa amplias zonas según un elemento definitorio y a partir de estas zonas continuar el proceso de división, según otros elementos, hasta llegar a unidades que se consideren caracterizadas. La segunda alternativa, y es también en la que se basa este artículo, consiste en realizar la selección de elementos esenciales, es decir, las variables más significativas y/o determinantes que definan las características de la unidad (VV.AA., 2000).

Basinski (1978) utilizó la idea de los factores limitantes, excluyentes o determinantes en todo el proceso metodológico de la planificación integrada. A este respecto, conviene tener en cuenta la posibilidad de la existencia de factores que proporcionan por si mismos todo el significado de la unidad, cuya presencia o ausencia impide el desarrollo de la actividad o la determina por completo, con independencia del significado de otros factores; es decir, condiciona de tal manera su vocación que frente a este pierden relevancia los demás (Spangle, 1976). Estas unidades reciben el nombre de unidades de síntesis y son las que proporcionan una mayor economía de medios en la integración y su posterior tratamiento (Gómez Orea, 2007).

La clasificación de unidades de síntesis definida por criterios ecológicos propuesta en este artículo se basa en la utilización de las variables de hidrografía, vegetación y fauna silvestre. Asimismo, se apoya en aspectos normativo-legales $y$ en una serie de clasificaciones, que identifican unidades en el territorio de forma sectorizada y definidas por cada una de las variables mencionadas. Cada uno de estos aspectos y clasificaciones se mencionan y se describen a continuación.

El Estado peruano, mediante la Ley General del Ambiente en su Artículo 99, establece una serie de unidades como ecosistemas frágiles para los cuales se adoptan medidas de protección especial, tomando en cuenta sus características y recursos singulares; y su relación con condiciones climáticas especiales y con los desastres naturales. Estas unidades están determinadas por las variables de hidrografía, vegetación y fauna silvestre, comprendiendo entre otras, desiertos, tierras semiáridas, montañas, pantanos, bofedales, bahías, islas pequeñas, humedales, lagunas alto andinas, lomas costeras, bosques de neblina y bosques relicto (Ley $\mathrm{N}^{\circ} 28611$, 2005).

En referencia a la hidrografía se tiene que la Secretaría de la Convención de Ramsar (2006) establece el Sistema de Clasificación de Tipos de Humedales de Ramsar, cuyo objetivo es asegurar la conservación y uso racional de los humedales ya que estos son de gran importancia para mantener los 
procesos ecológicos fundamentales debido a su rica flora y fauna y a los beneficios que reportan a las comunidades locales y a la sociedad humana en general. Se definen como zonas donde el agua es el principal factor controlador del medio y la vida vegetal y animal asociada a él. Se identifican 42 tipos de humedales, agrupados en tres categorías: marinos y costeros, continentales y artificiales. Los criterios utilizados, para declarar la importancia del sitio, están relacionados a la representatividad, rareza, unicidad y diversidad biológica.

Asimismo en materia normativo-legal, la ley de Recursos Hídricos, en el artículo 6, indica que las unidades reconocidas como bienes asociados al agua son los cauces o álveos, lechos y riberas de los cuerpos de agua, la vegetación ribereña y de las cabeceras de cuenca, las fajas marginales y las áreas ocupadas por los nevados y los glaciares; entre otras (Ley N²9338, 2009).

Para la vegetación se tiene que la clasificación de los sistemas ecológicos terrestres aporta un mapeo de unidades para la planificación de la conservación. Estas unidades se definen como grupos de comunidades vegetales que tienden a co-ocurrir en el paisaje debido a su relación con factores comunes y determinantes como procesos ecológicos, sustratos y/o gradientes ambientales. Los factores que determinan estas unidades están basados en los procesos ecológicos, el patrón espacial y la cobertura vegetal actual. La vegetación tiene un rol dominante en este enfoque, pues por un lado su expresión fisonómica o estructural es captada por los sensores remotos que son la fuente de información necesaria para el mapeo de grandes áreas y por otro lado refleja la respuesta biológica a los diferentes procesos ecológicos y características ambientales que se dan en un sitio especifico (Josse et al., 2007).

Bird Life International a través de su programa de Áreas Importantes para las Aves ${ }^{2}$ identifica, monitorea y protege una red global de IBAs para la conservación de las aves del mundo. Para ello establece una serie de criterios ornitológicos cuantitativos de selección, basados tanto como sea posible, en el conocimiento más exacto y actualizado de la distribución de las especies, tamaños y tendencias de las poblaciones (Birdlife, 2005).

La Ley de Áreas Naturales Protegidas establece los objetivos y/o criterios para determinar los espacios continentales y/o marinos del territorio nacional peruano, para conservar la diversidad biológica $\mathrm{y}$ demás valores asociados de interés cultural, paisajístico y científico, así como por su contribución al desarrollo sostenible del país. Para hacer efectivo el

${ }^{2}$ En ingles, Important Bird Areas (IBAs). establecimiento de un Área Natural Protegida esta debe tener claramente identificado el objetivo de su creación, que puede ser la protección de un ecosistema con nula o poca intervención humana, a fin de mantener inalterables los procesos ecológicos, especies o ecosistemas; o el mantenimiento de servicios ambientales que el área provee a la población. Es decir, el área debe contener valores biológicos, ecológicos, ambientales y/o culturales que ameriten la importancia de reconocerla como una zona prioritaria para su conservación, tales como representatividad, rareza, unicidad, contener especies de flora y fauna silvestre, en especial aquellas de distribución restringida o amenazadas; entre otros criterios (Ley No 26834, 1997).

\section{Materiales y métodos.}

El territorio peruano, orográficamente se debe considerar dividido por dos grandes manifestaciones, la Cordillera de los Andes y la Amazonía (Pulgar Vidal, 1987). Los Andes dividen longitudinalmente el país, en dirección Sureste a Noroeste, originando tres vertientes bien definidas, la vertiente del Pacífico, la del Atlántico y la del Titicaca (ONERN, 1980). La Amazonía conforma una semillanura cubierta de bosques, en donde el viento y las lluvias han creado un complejo sistema de bajas colinas y oteros que distribuyen las aguas pluviales entre los distintos ríos y contribuyen a dar al relieve diversas pendientes (Pulgar Vidal, 1987).

El Perú es un país mega-diverso pues, contiene 87 de las 117 zonas de vida del planeta (INRENA, 1994). Se compone de 11 ecorregiones $^{3}$, las cuales delimitan las características físicas y biológicas del territorio. Estas se estructuran de la siguiente forma (Figura 1): dos corresponden al mar, el Mar tropical y el Mar frío de la corriente Peruana; en la vertiente occidental se encuentra el Desierto del Pacífico, el Bosque Seco Ecuatorial, el Bosque Tropical del Pacífico, el Páramo y la Serranía Esteparia; en los territorios andinos por encima de los $3800 \mathrm{msnm}$ se ubica la Puna; por último, en la vertiente oriental se hallan tres ecorregiones, la Selva Alta, la Selva Baja y la Sabana de Palmeras (Brack \& Mendiola, 2000).

El procedimiento metodológico desarrollado en este artículo, para la obtención de la tipología de unidades de síntesis con criterios ecológicos, está basado en un enfoque descriptivo y conformado por cinco etapas, que se presentan a continuación (Figura 2):

${ }^{3}$ Áreas geográficas que se caracterizan por contar con similares condiciones climáticas, de suelo, hidrológicas, floristas y faunísticas, en estrecha interdependencia, perfectamente delimitables y distinguible de otras, además de gran utilidad práctica. 


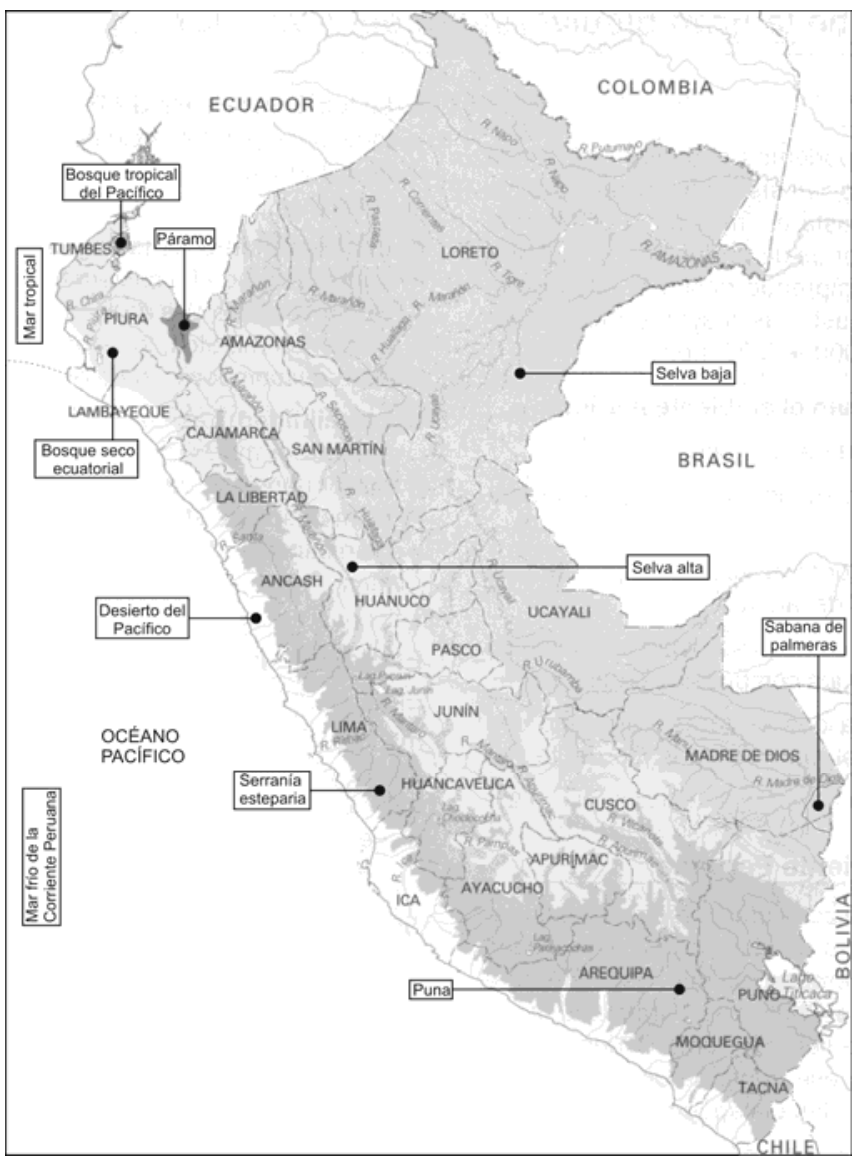

Figura 1. Las once ecorregiones del Perú (Fuente: Adaptado de Brack \& Mendiola, 2000).

Etapa (I). Uso de la información temática correspondiente a la hidrografía, vegetación y fauna silvestre. Esta es combinada mediante el método de superposición obteniéndose un mapa compuesto por los diferentes elementos o atributos que caracterizan cada variable. Esto se realiza a nivel meso, es decir a escala igual a 1:100 000.Cabe indicar que los atributos de cada variable ocupan un espacio en el territorio, cuando existe un solapamiento entre estos la hidrografía ejerce un rol dominante sobre la vegetación y la fauna silvestre marcando la unidad, como se muestra en los resultados, de la misma forma ocurre con la vegetación sobre la fauna silvestre.

Etapa (II). Análisis de cada variable según su criterio dominante. Los criterios utilizados para la definición de cada unidad no son excluyentes y de la misma forma puede suceder que no concurran todos.

En la hidrografía se han tomado en cuenta principalmente cuatro criterios:

(1) las diferentes formas en las que se presenta el agua para describir y clasificar el territorio,

(2) la velocidad de intercambio de nutrientes que se da en estas,
(3) la presencia en otras clasificaciones,

(4) y el reconocimiento normativo-legal de las mismas.

Los criterios que marcan la dominancia de la vegetación están representados por:

(1) los diferentes tipos y estructuras de cobertura vegetal actuales (caracterizadas por sus cualidades directas como endemismo, fragilidad y riqueza específica),

(2) los procesos ecológicos que las integran (tales como nivel de degradación (remanencia), estabilidad y potencial de transformación),

(3) la presencia en otras clasificaciones,

(4) y el reconocimiento normativo-legal de las mismas.

Los tipos de unidades asociados a la fauna silvestre se obtienen teniendo en consideración los siguientes criterios:

(1)la distribución de las especies (endemismo),

(2)los tamaños(abundancia y amenaza),

(3)las tendencias de las poblaciones,

(4)el reconocimiento en otras clasificaciones,

(5)y el carácter normativo-legal de las mismas.

Etapa (III). Caracterización de los sistemas. Empleando cada variable, y concretamente aquellos atributos y/o elementos que la hacen determinante, se definen aquellos sistemas que están presentes en el territorio. La hidrografía queda definida por los cuatro criterios mencionados, obteniéndose tres sistemas, el sistema fluvial con formas lineales y con velocidad de intercambio de nutrientes alta; las zonas húmedas o humedales de aguas tranquilas y formas poligonales; y el tercer sistema representado por los glaciares debido a su importancia como unidad a preservar y conservar.

Los sistemas asociados a la vegetación se dividen en dos, el primero referido a los ecosistemas arbóreos y el segundo a los arbustivos y/o herbáceos caracterizados ambos por sus cualidades directas, como endemismo, fragilidad, nivel de degradación (remanencia), estabilidad y riqueza específica; y sus cualidades indirectas como el potencial de transformación, el interés científico y/o educativo y la legislación aplicable con carácter de conservación.

En cuanto a la fauna encontramos tres sistemas, las áreas de importancia para la conservación de las aves, los hábitats con endemismos, y las áreas naturales protegidas. Estos pueden hacer referencia a la asociación de las especies con un determinado tipo de hábitat, o a su presencia en otras clasificaciones y/o a su reconocimiento normativo-legal. No obstante, 
todos están determinados por sus cualidades de abundancia (amenaza), endemismo e interés científico y/o educativo.

Etapa (IV). Caracterización de los sub-sistemas. Los sistemas incluyen unidades de menor complejidad, denominados sub-sistemas que se

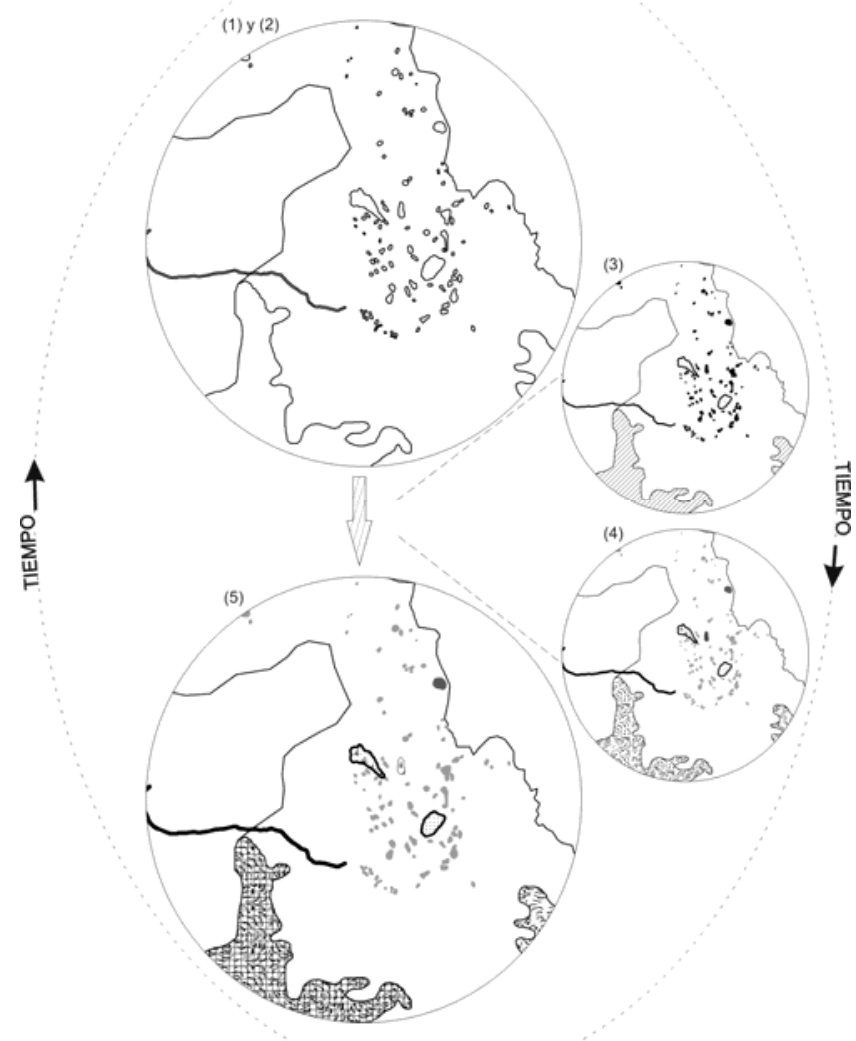

Figura 2. Procedimiento metodológico para la definición de las unidades de síntesis mediante la información temática (Fuente: elaboración propia).

determinan mediante la utilización de los diferentes criterios mencionados en la etapa anterior. Estos son el paso inmediato hacia la definición de las unidades.

Etapa (V). Obtención de las unidades. Por último, cada sub-sistema está, a su vez, compuesto por sus respectivas unidades de síntesis.

\section{Resultados y discusión.}

En la Tabla 1(ver anexo) se muestra en forma de resumen la tipología de unidades de síntesis para el territorio peruano que se pueden considerar para los procesos de meso ZEE. La tipificación está estructurada en cuatro niveles, un primer nivel correspondiente a la variable dominante, seguido de los sistemas asociados a esta, un tercer nivel compuesto por los sub-sistemas y, por último, el nivel conformado por las unidades de síntesis.

A continuación se describe a detalle la tipología mostrada en la Tabla 1.

I. Unidades de síntesis definidas por la variable hidrográfica.

I.1.Sistemas fluviales bien definidos

Los ríos son sistemas naturales enormemente dinámicos y complejos. Su principal función es el transporte de agua, sedimentos y nutrientes, pero además conforman espacios lineales de gran valor ecológico, paisajístico y territorial. En dinámica natural los ríos o sistemas fluviales cuentan con un espacio propio que ha sido modelado por las crecidas y que está conformado por el cauce, el corredor ribereño y, total o parcialmente, la llanura de inundación. Sus dimensiones han sido definidas por los principales eventos de crecida a los que el río ha asistido, denominándose a este espacio como territorio fluvial (Ollero, 2007).

En el Perú la gran mayoría de los ríos nacen en la parte alta de la Cordillera de los Andes, ecorregión de la Puna. Se tienen ríos de corto recorrido, fuerte pendiente y que transcurren en dirección uniforme de este a oeste, atravesando las ecorregiones de la Serranía Esteparia y el Desierto Costanero del Pacífico. Por otro lado, en la vertiente del Atlántico, los ríos son de largo recorrido de desnivel gradual y curso variado debido a la accidentada fisiografía del terreno, cruzan las ecorregiones de Selva Alta y Bosque Tropical Amazónico. Por último, la vertiente del Titicaca con ríos de mediana longitud de recorrido, pendiente reducida y cauces anchos y profundos que discurren por la ecorregión de la Puna y los Altos Andes. Estos cursos de agua son alimentados principalmente por las precipitaciones estacionales que ocurren en la parte alta y del deshielo de los nevados (ONERN, 1980).

La ley y el Reglamento de recursos hídricos regulan el uso y gestión integrada del agua. En dicho Reglamento se establecen tres zonas normalizadas de los ríos: el cauce, la ribera y la faja marginal. La caracterización de la funcionabilidad de estas zonas define las siguientes unidades de síntesis:

\section{I.1.1. Espacio fluvial}

El espacio fluvial es necesario para preservar el río, entendido en su concepción más común. Está constituida por el cauce (con presencia continua o discontinua de agua) y las riberas (ACA, 2006). Incluye cascadas y cataratas. Asimismo, se puede distinguir dos tipos de unidades: 
I.1.1.a. Espacio fluvial con aguas reguladas. El caudal es regulado mediante alguna infraestructura de origen antrópico.

I.1.1.b. Espacio fluvial con aguas no reguladas. Comportamiento natural del río.

\section{I.1.2. Faja marginal}

Es el área reservada a la preservación correcta y plena del régimen de corrientes en caso de avenida, por tratarse de una zona con un elevado riesgo de inundaciones (ACA, 2006). Son los terrenos o áreas inmediatas superiores a las riberas, necesarios para la protección, el uso primario del agua, el libre tránsito, la pesca, caminos de vigilancia u otros servicios. De esta forma la unidad de este sub-sistema es:

\section{I.1.2.a. Unidad definida por la faja marginal}

\section{I.2. Zonas húmedas}

De acuerdo a la Convención Ramsar (2006), se entiende por zonas húmedas o humedales a las extensiones de marismas, pantanos y turberas, o superficies cubiertas de aguas, sean éstas de régimen natural o artificial, permanentes o temporales, estancadas o corrientes, dulces, salobres o saladas, incluidas las extensiones de agua marina cuya profundidad en marea baja no exceda de seis metros.

El estado peruano mediante la Ley General del Ambiente - Ley Nº 28611 en su Artículo 99 establece los humedales como ecosistemas frágiles y reconoce su importancia como hábitat de especies de flora y fauna, en particular de aves migratorias, priorizando su conservación en relación con otros usos.

No obstante, estas unidades de síntesis siguen sometidas a degradaciones referidas principalmente al desecado para la ampliación de la agricultura, los procesos de urbanización no planificados y el uso como depósitos de desechos urbanos y rurales. Sumado a estas amenazas se tiene que los humedales con alguna categoría de protección son muy escasos y no satisfacen las necesidades de conservación de las aves residentes y migratorias (ProNaturaleza, 2010).

Otro de los inconvenientes es que la extensión espacial de los humedales puede fluctuar a lo largo del tiempo en respuesta a los cambios hidrológicos, tales como pequeñas variaciones en los niveles de agua subterránea. Esto da lugar a problemas de demarcación de límites con fines de gestión y zonificación (Adam, 1992).

En este artículo, las unidades de síntesis asociadas a los humedales se clasifican en cuatro tipos según su naturaleza: (1) Humedales de la costa; (2) Humedales altoandinos; (3) Humedales de la llanura Amazónica; y (4) Embalses.

\section{I.2.1. Humedales de la Costa}

Según Wolansky (2009) los humedales costeros deben ser vistos como un componente clave del ecosistema total compuesto por la cuenca del río, el río, el estuario y las aguas costeras. Son áreas con una descarga interna muy lenta, que combinan ambientes acuáticos y terrestres en un sistema dinámico en el que el papel del agua es crucial (Coiacetto, 1996).

El valor de los humedales radica en su función como protectores de la costa contra la erosión y contra la pérdida de infraestructura y vidas humanas. Son los hábitats que sustentan la pesca estacional o perenne y son vitales para aves migratorias y residentes. Además, proporcionan servicios ecológicos que tienen beneficios socioeconómicos para la población humana, incluyendo, de acuerdo a la ubicación, combustible, forraje, materiales de construcción, la madera, la pesca y la protección de las embarcaciones comerciales y de recreo (Williams et al., 2007).

Los humedales de la Costa se encuentran comprendidos en las ecorregiones de Mar Frío de la Corriente Peruana, Mar Tropical, Bosque Tropical del Pacífico, Bosque Seco Ecuatorial, Desierto Costanero del Pacífico y Serranía Esteparia.

Utilizando el sistema de clasificación de humedales de la Convención Ramsar (2006) y adaptándolo a la Costa peruana se puede distinguir los diferentes tipos de hábitats (ProNaturaleza, 2010), que se sirven para ordenar la siguiente lista de unidades de síntesis del territorio:

I.2.1.a. Humedales Marinos y Costeros compuestos por: aguas marinas someras permanentes, en la mayoría de los casos de menos de seis metros de profundidad en marea baja; se incluyen bahías y estrechos; islotes rocosos, roquedales y acantilados; playas de arena o de guijarros; puntas; estuarios; bajos intermareales de lodo, arena o con suelos salinos; pantanos y esteros (zonas inundadas) intermareales; marismas; manglares, pantanos, bosques inundados o inundables mareales de agua dulce; lagunas costeras salobres y/o saladas; lagunas de agua entre salobre y salada con por lo menos una relativamente angosta conexión al mar; lagunas costeras de agua dulce; y lagunas deltaicas de agua dulce.

I.2.1.b. Humedales Costeros Continentales: lagos permanentes, estacionales y/o intermitentes salinos y/o salobres y/o alcalinos; y manantiales de agua dulce, oasis.

\section{I.2.2. Humedales Altoandinos}

Los humedales altoandinos incluyen a aquellos humedales y complejos de humedales que forman parte de los ecosistemas ubicados principalmente en la ecorregión del Páramo y en la de la Puna.

El principal bien que proveen los humedales altoandinos es la provisión de agua, almacenamiento y regulación de caudales, estabilización de suelos, la prevención de deslaves y derrumbes y el mantenimiento del equilibrio ambiental tanto por permitir la sobrevivencia de especies singulares de flora y fauna, como por la fijación de carbono y purificación atmosférica y estabilización del clima (RAMSAR \& EHHA, 2008). 
Conforman sistemas con una gran variedad de ambientes que de acuerdo con su tipo y origen pueden comprender las siguientes unidades de síntesis:

I.2.2.a. Lagos y lagunas permanentes, estacionales y/o intermitentes de agua dulce y/o salinos y/o salobres y/o alcalinos (de más de 8 ha);

I.2.2.b. Pantanos: permanentes de agua dulce; sobre suelos inorgánicos, con vegetación emergente en agua por lo menos durante la mayor parte del período de crecimiento; y con vegetación arbustiva.

I.2.2.c. Bofedales o turberas no arboladas de altura con vegetación abundante y compacta alimentados por agua de lluvia y/o deshielos. Son asociaciones de vegetales que tienen un gran potencial productivo que es casi exclusivamente utilizado para pastoreo de alpacas y un número limitado de ovinos, vacunos y otras especies de animales (Sotomayor, 1990).La producción alpaquera es la principal actividad socioeconómica presente en estas zonas. La incorrecta gestión está causando el sobrepastoreo (Tapia \& Flores, 1984).

I.2.2.d. Humedales geotérmicos. Son los referidos a zonas de aguas termales y geiseres. Estas unidades por su distribución y tamaño pueden cobrar mayor importancia a un nivel de micro ZEE.

\section{I.2.3. Humedales de la Llanura Amazónica}

Las principales unidades de síntesis identificadas en este apartado son los aguajales y las cochas, se ubican $y$ se distribuyen principalmente en la ecorregión del Bosque Tropical Amazónico.

I.2.3.a. Los aguajales son ecosistemas forestales húmedos, inundados permanentemente por aguas negras, cubiertos por una extraordinaria vegetación pantanosa, donde se desarrolla de forma dominarte la especie Mauritia flexuosa, también conocida por su nombre común como palmera del Aguaje (Ruiz \& Levistre, 2011).

Los diferentes aprovechamientos que se encuentran relacionadas con esta unidad de síntesis son la alimentación, salud, construcción de viviendas, elaboración de utensilios, etc. No obstante, la actividad más importante es la que se deriva de la venta del fruto del aguaje, que es fuente de empleo y de ingresos para un porcentaje elevado de la población sobre todo femenina, siendo este su único medio de subsistencia (Padoch, 1988).

I.2.3.b. Las cochas ${ }^{4}$ están comunicadas con el río y entre sí por un canal o caño. Las más antiguas reciben agua sólo en la época de creciente, están cubiertas de vegetación y son de tipo pantanoso. Las más recientes tienen el espejo de agua libre de vegetación flotante, al menos en gran parte (Brack \& Mendiola, 2000).

I.2.4. Embalses

Un embalse es una acumulación artificial de agua en un ambiente natural que, por lo general se

${ }^{4}$ En la Selva Baja son muy abundantes las lagunas, conocidas como cochas (del quechua cocha = laguna) o tipishcas. construye interceptando un curso de agua (presa o dique), aunque los hay "laterales", es decir fuera de cauce, que aprovechan depresiones naturales del terreno, hacia las cuales se conduce el agua por canales, tuberías o túneles (Adler, 2005).

En la actualidad la finalidad de los embalses es regular, almacenar y desviar las aguas de los ríos para la producción agrícola, el uso humano e industrial en las ciudades, la generación de electricidad y el control de inundaciones. También se están utilizando para la recreación, el turismo y la acuicultura (WCD, 2000).

Los principales embalses en el Perú se distribuyen en las ecorregiones del Páramo, Serranía Esteparia y la Puna.

La unidad encontrada asociada a este sistema es:

I.2.4.a. Unidad definida por embalses

I.3. Áreas de Glaciares

Un glaciar es una masa de hielo que se acumula sobre la superficie terrestre, tanto por compactación y recristalización del hielo o la nieve. Los glaciares por su naturaleza resultan ser agentes erosivos, y por tal razón deben fluir (AGI, 2011). En el territorio peruano los glaciares corresponden al tipo alpino, ubicados en la Cordillera de los Andes y se distribuyen en la ecorregión de la Puna. Los usos actuales en estas unidades son los deportes de montaña y el turismo.

La ley de recursos hídricos (ley $\mathrm{N}^{\circ}$ 29338) en su artículo 6 establece como bienes asociados al agua las áreas ocupadas por los glaciares. Tomando en cuenta lo que indica la ley se establece la unidad de síntesis respectiva:

I.3.1.a. Unidad definida por glaciares.

II. Unidades de síntesis definidas por la variable de vegetación.

II.1.Ecosistemas arbóreos

\section{II.1.1. Bosques Relictos}

La Ley General del Ambiente - Ley No 28611 en su Artículo 99 establece a los bosques relictos como ecosistemas frágiles debido a su reducida superficie y a la fuerte presión a la que son sometidos, principalmente por la extracción de leña, conversión en carbón, quema de pastos, sobre pastoreo y ampliación de la frontera agrícola. La importancia del ecosistema mismo (para proveer agua para la Costa), así como sus amplios recursos fitogenéticos y su potencial ecoturístico tienen un valor económico mucho mayor a la utilización efímera de zonas taladas para cultivos (Weigend et al., 2006).

Estas unidades de síntesis se han diferenciado en dos tipos, principalmente por su ubicación geografía y composición vegetal:

II.1.1.a. Los Bosques Relictos Altoandinos. Estos relictos de bosque son dominados por especies del género Polylepis, aunque en muchos lugares otras especies leñosas como Buddleja sp., Clethra sp., Gynoxys sp., Podocarpus sp. o Prumnopitys sp. también están presentes. Estos bosques cumplen un rol central en la ecología altoandina como hábitat para 
muchas especies de plantas y animales, y como importante fuente de recursos para los habitantes locales. Representan uno de los ecosistemas más amenazados del mundo (Kessler, 2006).

La distribución actual de los bosques de Polylepis es muy local. Existen algunas zonas con bosques relativamente extensos, pero en su gran mayoría los bosques son pequeños rodales restringidos a laderas rocosas y quebradas o matorrales abiertos en laderas montañosas (Kessler, 2006). Se distribuyen en la ecorregión de la Puna, principalmente en las zonas sur y centro del país.

II.1.1.b. Los Bosques Relictos Mesoandinos. Localizados en la vertiente occidental de los Andes, distribuidos en la ecorregión del Páramo en el norte de Perú, son hábitats con alta fitodiversidad y un índice de endemismo muy elevado, se encuentran fuertemente fragmentados (Weigend et al., 2005). Las fuertes pendientes, los suelos superficiales, afloramientos rocosos y gran pluviosidad califican los espacios donde se localizan como tierras de protección (MINAM, 2010).

\section{II.1.2. Bosques de Neblina}

La definición más ampliamente adoptada de los bosques nublados se refiere a "bosques que se encuentran frecuentemente cubiertos por nubes o niebla” (Stadtmüller, 1987). Estos bosques se distribuyen principalmente por encima de los $1000 \mathrm{~m}$ de altitud en la vertiente noroccidental de los Andes del Perú, en la ecorregión del Páramo.

Son ecosistemas que presentan una alta diversidad y endemismos, entre estos se encuentran especies de coníferas nativas pertenecientes a la familia Podocarpaceae que juegan un rol importante en la dinámica del ecosistema. Sin embargo, debido a la calidad de sus maderas están siendo extraídas de manera alarmante (Vicuña-Miñano, 2005).

Los estudios sobre el comportamiento hidrológico en las zonas donde se encuentran los bosques de neblina, indican que la deforestación puede influenciar de manera importante en la pérdida de agua. Esto ocurre porque se aumenta el nivel de evapotranspiración y se reduce y/o elimina la entrada adicional de agua en el bosque a través de la precipitación horizontal (Zadroga, 1981). También, la deforestación puede traer consecuencias graves para las cuencas bajas, como inundaciones, procesos acelerados de erosión y una disminución marcada de los caudales de los ríos durante las estaciones de estiaje (Stadtmüller, 1987).

La ley General del Ambiente - Ley No 28611, en su Artículo 99, establece a los bosques de neblina como ecosistemas frágiles. La unidad coincide con la clasificación del sub-sistema y es:

II.1.2.a. Unidad definida por bosques de neblina

II.1.3. Bosques Tropicales Estacionalmente Secos - BTES
El bosque tropical estacionalmente seco está formado por árboles caducifolios y muchas plantas epifitas; la sabana, conformada por árboles dispersos de sapote, faique y algarrobo; y el algarrobal, con predominancia del algarrobo (Brack \& Mendiola, 2000). Consecuentemente los procesos ecológicos son moderadamente estacionales y la productividad primaria neta es menor que en los bosques húmedos, por presentarse solo en la temporada de lluvias (Pennington et al., 2000).

Estos bosques son importantes por su diversidad florística exclusiva en el continente americano, además por proveer una serie de productos diferentes a la madera (fibras, látex, goma, resinas, frutos, forraje, etc.) y como captadores y reguladores del recurso hídrico (MINAM, 2010).

Los BTES están sujetos a múltiples presiones. Las principales causas de pérdida de estos bosques son el cambio climático, la fragmentación del bosque, los incendios y la ampliación de la frontera agrícola, que se presentan a menudo simultáneamente (Miles et al., 2006).

Linares-Palomino (2004) clasificó los BTES del Perú en tres subunidades biogeográficas, las cuales se han utilizado para definir las unidades de síntesis:

II.1.3.a. Unidad de BTES Ecuatoriales, distribuidas principalmente en la ecorregión del Páramo.

II.1.3.b. Unidad de BTES interandinos (Sistema del río Huancabamba, Sistema del río Marañón, Sistema del río Mantaro, Sistema del río Apurímac y otros remanentes menores), presentes en la ecorregión de la Selva Alta (Yungas).

II.1.3.c. Unidad de BTES orientales, distribuidos en la ecorregión de la Selva Alta (Yungas).

\section{II.1.4. Bosques Amazónicos}

La Amazonía peruana (parte andina, selva alta y selva baja), comprende una gran masa de bosques, con núcleos o biomasa de bosques húmedos, mesofíticos y xerofíticos, y una gran variedad de tipos relacionados con la localidad (altitud y latitud), donde se desarrolla una compleja diversidad de plantas clasificables y diferenciadas por sus portes y estructura en alturas, variabilidad diamétrica de sus troncos y estratos, y grados de asociación en frecuencia y densidad (IIAP, 2004).

La complejidad para la caracterización de las formaciones o comunidades vegetales en la parte del llano amazónico, como en la región andina, requiere de mayores exploraciones y muestreos botánicos sistematizados y georeferenciados (IIAP, 2004).

Para la definición de las unidades de síntesis que definen este sub-sistema se ha adaptado la clasificación de los sistemas ecológicos de la cuenca amazónica (Josse et al., 2007), la misma que se menciona a continuación:

II.1.4.a. Bosques húmedos andinos de Yungas (ecorregión de Selva alta). 
II.1.4.b. Bosques húmedos amazónicos (ecorregión de Selva baja).

II.1.4.c. Bosques secos y matorrales xéricos andinos de Yungas (ecorregión de Selva alta).

II.2. Ecosistemas arbustivos y/o herbáceos

II.2.1. Matorrales

Compuestos por vegetación secundaria que sustituye a bosques y arbustos como consecuencia de la acción humana. En el extremo árido la distribución se da en la transición o se combina con elementos de ecosistemas del desierto peruano. Se trata de un mosaico de matorrales y comunidades de gramíneas muy xeromórficas con afinidad florística andina (NatureServe, 2009), distribuidos en la ecorregion de la Serranía Esteparia.

Este sub-sistemase halla conformado por dos sistemas ecológicos terrestres endémicos y otro no endémico, pero de distribución restringida y sin protección (Véliz et al., 2008), los cuales definen las unidades de síntesis siguientes:

II.2.1.a. Matorrales desérticos montanos y basimontanos. Ubicados en laderas montañosas de pendiente elevada, entre los 1500 y 3000 msnm aproximadamente entre el norte de Lima y el sur de Ancash.

II.2.1.b. Matorrales altimontanos de la puna xerofítica, característicos de las alturas del sur del Perú ubicados a partir de los 3000 msnm.

\section{II.2.2. Lomas Costeras}

Ono (1986) se refiere a los ecosistemas de lomas costeras como "praderas de vegetación en medio del desierto”. Son unidades fitogeográficas periódicas que habitualmente contienen un número elevado de géneros y especies endémicas (Mostacero et al., 1996).

La vegetación de lomas se puede desarrollar en las laderas orientadas hacia el mar, hasta los $1000 \mathrm{msnm}$, con variaciones a nivel local. Encima de los 1000 msnm cesa la influencia de las neblinas debido al fenómeno de inversión térmica, no existiendo vegetación invernal allí (Brack \& Mendiola, 2000). Principalmente, se distribuyen en el centro y sur de la ecorregión del Desierto Costanero del Pacifico.

Las lomas costeras debido a que son "islas de vegetación” en el desierto, han tenido gran importancia económica y ambiental, ya que proporcionaban leña, forraje y madera, atenuando las condiciones de extrema aridez del desierto costero (Engel, 1981). Debido al aprovechamiento insostenible (sobrepastoreo y deforestación) se ha generado una dramática disminución de estos ecosistemas (Mostacero et al., 1996). Actualmente, esta disminución también se da por la presión urbanística, como es el caso de la ciudad de Lima.

En la legislación, Ley General del Ambiente - Ley $\mathrm{N}^{\circ}$ 28611, en su Artículo 99 se establece a las lomas costeras como ecosistemas frágiles, por lo que se deben adoptar medidas de protección especial, tomando en cuenta sus características y recursos singulares; y su relación con condiciones climáticas especiales y con los desastres naturales. La unidad asociada a este sub-sistema es:

II.2.2.a. Unidad definida por lomas costeras

\section{II.2.3.Cardonales}

Son sistemas compuestos por vegetación climácica abierta, dominados por grandes cactáceas columnares arborescentes intercaladas con otras del tipo arbustivas y globulares, así como matorrales muy xeromórficos. Se desarrolla en las serranías y laderas andinas occidentales (NatureServe, 2009), correspondiente a las ecorregiónes del Bosque Seco Ecuatorial, el Desierto Costanero y la Serranía Esteparia.

Según la clasificación de los sistemas ecológicos ${ }^{5}$ (Josse et al. 2003), en el territorio peruano se localizan tres sistemas de cardonales y matorrales desérticos, considerados de importancia ecológica, dos tienen carácter endémico y otro esta dominado en el estrato superior por la cactácea arborescente Browningiacandelaris, endémica regional. Estos componen las unidades de síntesis de este subsistema:

II.2.3.a. Cardonales y matorrales desérticos del piedemonte occidental de la puna húmeda.

II.2.3.b. Cardonales y matorrales montanos desérticos.

II.2.4. Pajonal

Sub-sistema que incluye varias asociaciones de herbazales, en general dominados por especies robustas de gramíneas de crecimiento amacollado y a menudo hojas duras o pungentes. Se hallan ampliamente afectados por el uso ganadero, principalmente camélidos andinos y bovinos. Ubicados en laderas montañosas con suelos bien drenados (NatureServe, 2009).Se distribuyen principalmente en las ecorregiones de la Serranía costanera, el Páramo, la Selva alta y la Puna. Adaptando la clasificación de los sistemas ecológicos (Josse et al. 2003), se derivan las siguientes unidades de síntesis:

II.2.4.a. Pajonal altimontano y montano paramuno

II.2.4.b. Pajonal altoandino y altimontano de la Puna

II.2.4.c. Pajonal arbustivo altoandino y altimontano de Yungas

II.2.5. Ecosistemas Dunares del Desierto Costero

Las dunas son ecosistemas muy dinámicos y variables cuyo funcionamiento se explica por la interrelación entre arenas móviles y vientos constantes y regulares. Las dunas litorales se presentan, por lo general, como largas franjas paralelas a la orilla, cuya abundancia, altura y ancho, están condicionadas por el sustrato y el clima. Las dunas continentales son

\footnotetext{
${ }^{5}$ Los sistemas ecológicos son comunidades de plantas que se localizan en paisajes con sustratos y/o gradientes ambientales similares.
} 
muy variadas en su forma, pueden aparecer aisladas en regiones rocosas o en campos dunarios de gran extensión (Müller-Hohenstein, 1992).

En el Perú se distribuyen en la ecorregión del Desierto costanero. Las actividades asociadas a esta unidad son los deportes de riesgo y el turismo. Las diferencias existentes en la geología histórica, la sucesión paisajística, la gestión y el clima, traen como consecuencia varios tipos de zonificación de los ecosistemas dunares (Doing, 1995). La unidad identificada es la siguiente:

II.2.5.a. Unidad definida por dunas

III. Unidades de síntesis definidas por la variable de fauna

III.1. Áreas de Importancia para la Conservación de las Aves

Las Áreas Importantes para la Conservación de las Aves (IBAs) son sitios críticamente importantes a nivel mundial para las aves y la biodiversidad. Cada área cuenta con la suficiente extensión para mantener poblaciones viables de la mayoría de especies posibles para las cuales fue identificado; estas zonas se distribuyen por todo el Perú, sumando en la actualidad un total de 190. Casi la totalidad de las 87 especies globalmente amenazadas presentes en Perú se encuentran cubiertas por las IBAs (Birdlife, 2005).

Este sistema queda representado a través de la siguiente unidad de síntesis:

III.1.1.a. Unidad definida por las Áreas de Importancia para la Conservación de las Aves

\section{III.2. Hábitats con endemismos}

El Perú cuenta con diferentes áreas con endemismos que se encuentran fuera del Sistema Nacional de Áreas Protegidas, distribuidas por las diferentes ecorregiones del territorio peruano y abarcando especies de aves, peces continentales, anfibios, mamíferos, moluscos terrestres y fanerógamas (Lleellish et al., 2005). Estos hábitats conforman la unidad:

III.2.1.a. Unidad definida por Hábitats con endemismos

III.3. Áreas Naturales Protegidas

Son áreas protegidas mediante la ley $\mathrm{N}^{\circ} 26834$, debido a su importancia para la conservación de la diversidad biológica y demás valores asociados de interés cultural, paisajístico y científico, así como por su contribución al desarrollo sostenible.

III.3.1. Áreas Naturales Protegidas de Uso Indirecto

Son aquellas que permiten la investigación científica no manipulativa, la recreación y el turismo, en zonas apropiadamente designadas y manejadas para ello. En estas áreas no se permite la extracción de recursos naturales, así como modificaciones y transformaciones del ambiente natural. Las unidades de síntesis definidas son:

III.3.1.a. Parques Nacionales.

III.3.1.b. Santuarios Nacionales.
III.3.1.c. Santuarios Históricos.

III.3.2. Áreas Naturales Protegidas de Uso Directo

En estas áreas se permite el aprovechamiento o extracción de recursos, prioritariamente por las poblaciones locales, en aquellas zonas y lugares y para aquellos recursos, definidos por el plan de manejo del área. Otros usos y actividades que se desarrollen deberán ser compatibles con los objetivos de la misma. Las unidades de síntesis definidas son:

III.3.2.a. Reservas Nacionales.

III.3.2.b. Reservas Paisajísticas.

III.3.2.c. Refugios de Vida Silvestre.

III.3.2.d. Reservas Comunales.

III.3.2.e. Bosques de Protección.

III.3.2.f. Cotos de Caza.

III.3.2.g. Áreas de Conservación Regional.

\section{Conclusiones.}

La unidad de síntesis se debe considerar como un sistema que incluye una serie de sub-sistemas. En el presente artículo se han caracterizado ocho sistemas: tres asociados a la hidrografía, dos a la vegetación y otros tres a la fauna. De igual manera estos sistemas incluyen un total de veinte sub-sistemas, que se desagregan a su vez en cuarenta y tres unidades de síntesis definidas por criterios ecológicos.

Esta tipificación tiene la intención de servir como referencia para los procesos de meso ZEE, pero considerando la complejidad del territorio, intenta ser flexible y estar abierta a nuevas interpretaciones y ampliaciones con nuevas unidades que no se hayan considerado en esta primera aproximación. Por lo tanto, la clasificación realizada no es excluyente.

La utilización de las unidades de síntesis con criterios ecológicos debe ser complementada con unidades marcadas por otros criterios como por ejemplo productivos, histórico-culturales, urbanoindustriales, etc., para facilitar de esta forma el análisis integral del territorio. Si la elección de las unidades de síntesis se realiza con acierto puede coadyuvar al proceso de planificación del territorio y garantizar que se logre con éxito la asignación de recomendaciones de uso respectivas.

La clasificación desarrollada en este artículo se apoya en los alcances conseguidos en otras clasificaciones sectoriales como la Convención de Ramsar para la conservación y uso racional de los humedales; los sistemas ecológicos terrestres de NatureServe para la planificación de la conservación de la vegetación; las áreas de importancia para las aves (IBAs) de Bird Life International. Asimismo, se tiene muy en cuenta que las unidades clasificadas ocupan un espacio en la legislación conservacionista del país debido a su importante valor ecológico.

En conjunción con lo expresado por el IIAP (2004), para la definición de unidades asociadas a los bosques amazónicos, debido a la complejidad para la caracterización de las formaciones o comunidades 
vegetales, es recomendable realizar una mayor precisión en el análisis, siendo una alternativa la clasificación realizada por la NatureServe de los sistemas ecológicos de la cuenca amazónica. Esta clasificación ha servido de ayuda para definir las unidades presentes en el sub-sistema de bosques amazónicos, en el de cardonales y en el de pajonal.

Cabe indicar que las Áreas Naturales Protegidas (ANP) atienden a criterios socioeconómicos por su estado normativo-legal, no obstante se incluyen en este artículo debido a que el motivo de su reconocimiento se debe de forma notable a su importancia ecológica. Es por ello que en la ZEE se las agrupa en el sub-modelo de valor bio-ecologico y se zonifican como zonas de protección y conservación ecológica (DGOT, 2011).

Asimismo, estas se han considerado dentro de la tipología definida por la fauna, debido a que la localización de los vertebrados está fuertemente ligada en unos casos, a los tipos de formaciones vegetales y de cubierta; en otros, a la presencia de agua. No obstante, cabe señalar que estas unidades también han podido ser consideradas dentro de la variable de vegetación, ya que en estas la diversidad biológica se debe tanto a la riqueza en fauna como en flora.

Finalmente, para la definición de las unidades ecológicas económicas (UEE) de un territorio se recomienda utilizar, en primer lugar, el método de las unidades de síntesis, ya que de esta forma se logra reducir el número de polígonos a analizar mediante otros métodos; y en concordancia con lo expresado por Gómez Orea (2007), estas pueden constituirse en una menor inversión económica para el análisis del territorio, ofreciendo una alternativa para la meso ZEE en procesos donde el presupuesto es escaso o limitado.

\section{Literatura citada.}

Adam P. 1992. Wetlands and wetland boundaries: problems, expectations, perceptions and reality. Wetlands, 11(2): 60-67.

Adler F. 2005. Los embalses y los recursos hídricos superficiales. Revista CET, Nro.27. 11 p.

ACA - Agència Catalana de L'aigua. 2006. Directrius de planificació I gestió de l’espai fluvial. Guia Técnica. Barcelona, Generalitat de Catalunya. 53 p.

AGI - American Geosciences Institute. 2011. Glossary of Geology. Fifth Edition (revised). Edited by Klaus K.E. Neuendorf, James P. Mehl, Jr., and Julia A. Jackson. $800 \mathrm{p}$.

Basinski J.J. 1978. Land use of the South Coast of new South Wales. CSRIO, Melbourne. 129 p.

Birdlife International and Conservation International. 2005. Áreas Importantes para la Conservación de las Aves en los Andes Tropicales: sitios prioritarios para la conservación de la biodiversidad. Quito, Ecuador: BirdLife International (Serie de Conservación de BirdLife No. 14).

Brack A \& Mendiola C. 2000. Ecología del Perú. Programa de las Naciones Unidas para el Desarrollo (PNUD). Editorial Bruño. Lima-Perú. 496 p.
Coiacetto E. 1996. A model for use in the management of coastal wetlands. Landscape and Urban Planning, 36(1): 27-47.

Convención de Ramsar y Grupo de Contacto EHAA. 2008. Estrategia Regional para la Conservación y Uso Sostenible de Humedales Altoandinos. Gobiernos de Ecuador y Chile, CONDESAN y TNC-Chile.

Dansereau P. 1957. Biogeography an ecological perspective. The Royal Press, New York.

DGOT- Dirección General de Ordenamiento Territorial. 2011. Guía técnica de Modelamiento Temático para la Zonificación Ecológica Económica. Documento técnico. Lima, Perú. 95 p.

Doing H. 1995. Landscape ecology of the Dutch coast. Journal of Coastal Conservation 1: 145-172.

Engel F. 1981. Prehistoric Andean Ecology Man, Settlement and Environment in the Andes. The Deep South. University of New York. USA.

Gómez Orea D. 2007. Ordenación territorial. 2da edición. Ediciones Mundi-Prensa. 766 p.

González Bernáldez F. 1973. Estudio ecológico de la subregión de Madrid. COPLACO, Madrid.

Hills A. 1961. The ecological basis for land-use planning. Ontario Dept. of Lands and Forests, Research Branch. Toronto. 204 p.

IIAP - Instituto de Investigaciones de la Amazonía Peruana. 2004. Diversidad de la Amazonía Peruana expresada en un mosaico de imágenes satélite. Documento Técnico No. 12 Serie IIAP - BIODAMAZ. Iquitos, Perú.

INRENA - Instituto Nacional de Recursos Naturales. 1994. Mapa ecológico del Perú: Guía explicativa. Ministerio de Agricultura, Lima, Perú. 271 p.

Josse C., Navarro G., Comer P., Evans R., FaberLangendoen D., Fellows M., Kittel G., Menard S., Pyne S., Reid M., Schulz K., Snow K \& Teague J. 2003. Ecological Systems of Latin America and the Caribbean: A Working Classification of Terrestrial Systems. NatureServe, Arlington, VA.

Josse C., Navarro G, Encarnación F., Tovar A., Comer P., Ferreira W., Rodríguez F., Saito J., Sanjurjo J., Dyson J., Rubín de Celis E., Zárate R., Chang J., Ahuite M., Vargas C., Paredes F., Castro W., Maco J. \& Reátegui F. 2007. Sistemas Ecológicos de la Cuenca Amazónica de Perú y Bolivia. Clasificación y mapeo. NatureServe. Arlington, Virginia, EE UU. 94 p.

Kessler M. 2006. Bosques de Polylepis. Botánica Económica de los Andes Centrales. Editores: M. Moraes R., B. Øllgaard, L. P. Kvist, F. Borchsenius\& H. Balslev. Universidad Mayor de San Andrés, La Paz. 110-120.

Lleellish, M., Silva I., Martínez C. \& del Pozo P. 2005. Elaboración de criterios de cobertura geográfica para el establecimiento de áreas prioritarias para el desarrollo del biocomercio. Grupo Técnico Producto 4. Disponible en la web: http://www.ibcperu.org/doc/isis/11121.pdf (revisado 30/10/2012)

Linares-Palomino R. 2004. Los Bosques Tropicales Estacionalmente Secos: II. Fitogeografía y Composición Florística. Arnaldoa. 11(1): 103-138.

McHarg I. 1969. DesignwithNature. American Museum of Natural History [by] the Natural History Press, 197 p.

Miles L; Newton A; Defries R; Ravilious C; May I; Blyth S; Kapos V\&Gordon J. 2006. A global overview of the 
conservation status of tropical dry forests. J. Biogeog. 33: $491-505$.

MINAM - Ministerio del Ambiente. 2010. Mapa del patrimonio forestal nacional. $6 \mathrm{p}$.

Mostacero J., Mejía F \& Peláez F. 1996. Fitogeografía del Norte del Perú. Serie Ciencias-CONCYTEC. LimaPerú. 406 p.

Müller-Hohenstein K. 1992. Las dunas como ecosistemas: experiencias en Europa, Arabia y África. BOSQUE 13(1): 9-21.

NatureServe. 2009. International Ecological Classification Standard: Terrestrial Ecological Classifications. Sistemas Ecológicos de los Andes del Norte y Centro. NatureServe Central Databases. Arlington, VA.

ONERN - Oficina Nacional de Evaluación de Recursos Naturales. 1980. Inventario Nacional de Lagunas y Represamientos. Segunda Aproximación. Lima. 323 p.

Ollero A. 2007. Territorio fluvial. Diagnóstico y propuesta para la gestión ambiental y de riesgos en el Ebro y los cursos bajos de sus afluentes. Bilbao, Bakeaz /Fundación Nueva Cultura del Agua. 256 p.

Ono M. 1986. Definition, classification and taxonomic significance of the Lomas vegetation. In: M. Ono (editor) Taxonomic and Ecological Studies on the Lomas Vegetation in the Pacific Coast of Peru. Makino Herbarium, Tokyo Metropolitan University. TokyoJapan. p. 5-14.

Padoch C. 1988. Aguaje (Mauritia flexuosa L.f.) in the economy of Iquitos, Peru. Advances in Economic Botany 6: 214-224.

Pennington T., Prado D \& Pendry C. 2000. Neotropical Seasonally dry forests and quaternary vegetation changes. Journal of Biogeography, 27 (2): 261-273.

PRONATURALEZA - Fundación Peruana para la Conservación de la Naturaleza. 2010. Humedales en la costa peruana. 1a. edición. ProNaturaleza.

Pulgar Vidal J. 1987. Geografía del Perú. Las Ocho Regiones Naturales. 9a ed. Peisa. Lima. Perú. 244 p.

Ramos Fernández A.1979. Planificación física y ecología. Modelos y métodos. Madrid, EMESA.

Ruiz J \& Levistre J. 2011. El aguajal: el bosque de la vida en la Amazonía peruana. Ciencia Amazónica, 1(1): 31 40.

Secretaría de la Convención de Ramsar. 2006. Manual de la Convención de Ramsar: Guía a la Convención sobre los Humedales (Ramsar, Irán, 1971), 4a. edición. Secretaría de la Convención de Ramsar, Gland (Suiza).

Sotomayor M. 1990. Tecnología Campesina en el pastoreo altoandino. Cooperación Técnica del Gobierno Suizo, Lima. 143 p.

Spangle W \& Associates. 1976. Earth-science information in land-use planning; guidelines for earth scientists and planners. U.S. Geological Survey, Circular 721, Washington, $28 \mathrm{p}$.

Stadtmüller T. 1987. Cloud Forests in the Humid Tropics: A Bibliographic Review. The United Nations University, Tokyo and Centro Agronómico Tropical de Investigación y Enseñanza, Turrialba, Costa Rica, 81 p.

Tapia M. \& Flores J. 1984. Pastoreo y Pastizales de los Andes del Sur del Perú. Instituto Nacional de Investigación y Promoción Agropecuaria. 312 p.

VV. AA. 2000. Guía para la elaboración de estudios del medio físico. Contenido y Metodología. Ed. Ministerio del Medio Ambiente.

Véliz C., Tovar L.A., Tovar C., Regal F. \& Vásquez P. 2008. ¿Qué áreas conservar en nuestras Zonas Áridas? Seleccionando sitios prioritarios para la conservación en la Ecorregión Desierto de Sechura - Perú. Zonas Áridas 12(1): 36-59.

Vicuña-Miñano E. 2005. Las podocarpáceas de los bosques montanos del noroccidente peruano. Revista Peruana de Biología. 12(2): 283-88.

Weigend M., Rodríguez-Rodríguez E. \& Arana C. 2005. The relict forests of Northwest Peru and Southwest Ecuador. En: M. Weigend, E. Rodríguez \& C. Arana (comp.), Los Bosques Relictos del NO de Perú y SO de Ecuador. Rev. peru. biol. 12(2): 189-194.

Weigend M. Dostert N. \& Rodríguez-Rodríguez E. 2006. Bosques relictos de los Andes peruanos: Perspectivas económicas. Botánica Económica de los Andes Centrales. Editores: M. Moraes R., B. Øllgaard, L. P. Kvist, F. Borchsenius \& H. Balslev. Universidad Mayor de San Andrés, La Paz. 130-145.

Williams M. J., Coles R. \& Primavera J.H. 2007. A lesson from cyclone Larry: An untold story of the success of good coastal planning. Estuar. Coast. Shelf Sci. 71, 364367.

Wolanski E. 2009. Coastal Wetlands: A Synthesis. In: Coastal Wetlands: An Integrated Ecosystem Approach, editors: Gerardo M. E. Perillo, Eric Wolanski, Donald R. Cahoon, Mark M. Brinson. Elsevier. p. 1 - 62.

WCD - World Commission on Dams. 2000. Dams and development a new framework for decision making. Earthscan Publications Ltd, London and Sterling, VA. $404 \mathrm{p}$.

Zadroga F. 1981. The hydrological importance of a montane cloud forest area of Costa Rica. In: Lal R. and Russel E.W. (Eds.) Tropical Agricultural Hydrology. John Wiley \& Sons Ltd. p.59-73.

Zonneveld I. 1989. The land unit - A fundamental concept in landscape ecology, and its Applications. Landscape Ecology, 3(2): 67-86. 


\section{ANEXO}

Tabla 1. Tipología de unidades de síntesis para el territorio peruano (Fuente: elaboración propia).

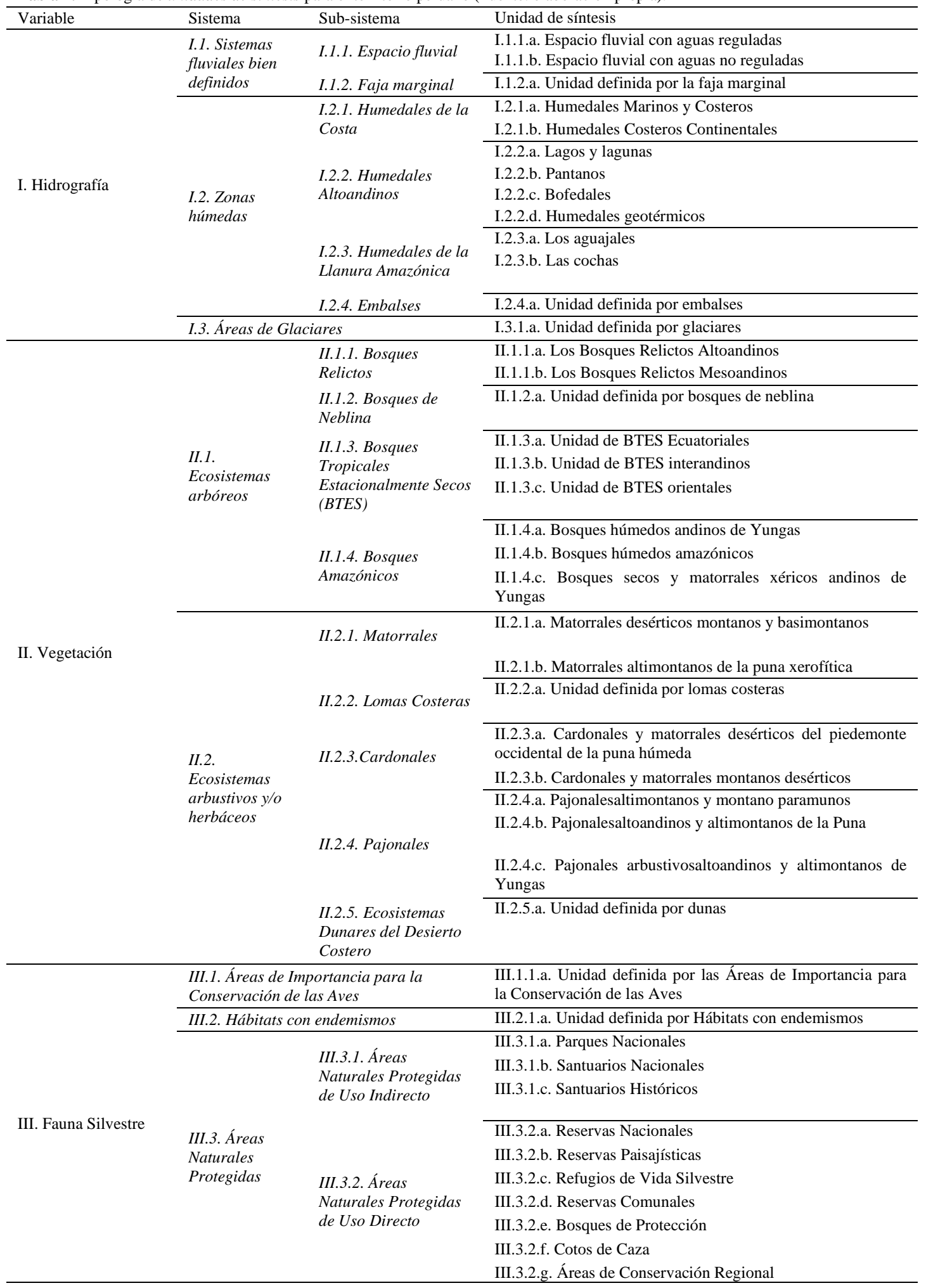

${ }^{1}$ Universidad Nacional Agraria La Molina, Lima - Perú. Correo electrónico santiagomadrigal@lamolina.edu.pe 